\title{
Growth Rates in the Quaquaversal Tiling*
}

\author{
B. Draco, ${ }^{1}$ L. Sadun, ${ }^{1}$ and D. Van Wieren ${ }^{2}$ \\ ${ }^{1}$ Department of Mathematics, University of Texas, \\ Austin, TX 78712, USA \\ \{brim, sadun\}@math.utexas.edu \\ 2 Department of Computer Science and Engineering, \\ Pennsylvania State University, \\ University Park, PA 16802, USA \\ dvw@buchni.bk.psu.edu
}

\begin{abstract}
Conway and Radin's "quaquaversal" tiling of $\mathbb{R}^{3}$ is known to exhibit statistical rotational symmetry in the infinite volume limit. A finite patch, however, cannot be perfectly isotropic, and we compute the rates at which the anisotropy scales with size. In a sample of volume $N$, tiles appear in $O\left(N^{1 / 6}\right)$ distinct orientations. However, the orientations are not uniformly populated. A small $\left(O\left(N^{1 / 84}\right)\right)$ set of these orientations account for the majority of the tiles. Furthermore, these orientations are not uniformly distributed on $S O$ (3). Sample averages of functions on $S O(3)$ seem to approach their ergodic limits as $N^{-1 / 336}$. Since even macroscopic patches of a quaquaversal tiling maintain noticeable anisotropy, a hypothetical physical quasicrystal whose structure was similar to the quaquaversal tiling could be identified by anisotropic features of its electron diffraction pattern.
\end{abstract}

\section{Introduction and Results}

This paper concerns statistical properties of Conway and Radin's [CR] quaquaversal (QQ) tiling of Euclidean 3-space. A QQ tiling is made by copies of a single wedge-shaped tile, appearing in an infinite number of distinct orientations. The set of orientations is a dense subgroup of $S O(3)$, and the orientations are in fact uniformly distributed on $S O$ (3): Given a smooth function $f$ on $S O(3)$, the average value of $f$ on the orientations of tiles in a large patch of $\mathbb{R}^{3}$ is close to the integral of $f$ over $S O(3)$ (with respect to Haar measure). This property, called "statistical rotational symmetry" by Radin [R1],

* The work of L. S. was partially supported by NSF Grant No. DMS-9626698 and Texas ARP Grant 003658-152. 


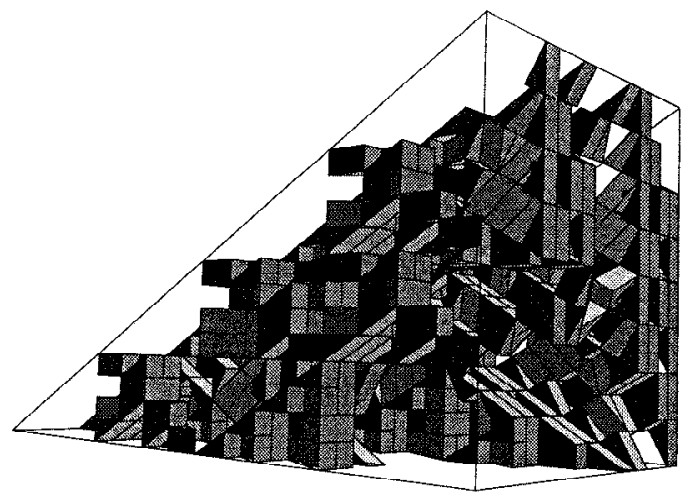

Fig. 1. Part of a QQ tiling.

suggests that a large quasicrystal based on the QQ tiling should appear round, and its physical properties should be isotropic. We call such a material "asymptotically round."

In this paper we address the question of how large such a quasicrystal would have to be to make its roundness manifest. We show that the convergence to rotational symmetry is polynomial in the sample size, but with an extremely small exponent. Samples with $10^{24}$ tiles have pronounced anisotropy, and even samples with $10^{100}$ tiles have noticable anisotropy. In short, the asymptotic roundness of the QQ tiling, while mathematically correct, is well beyond the range of any conceivable physics. If a physical material were modeled on the QQ tiling, it would exhibit considerably more isotropy than a crystal, while still being considerably less isotropic than a liquid or a glass. These comparisons are discussed further in Section 5. There is some evidence [R2] that such asymptotically round quasicrystals may indeed exist in nature.

The slow growth of complexity is not unique to the QQ tiling, but is a typical feature of three-dimensional substitution tilings. Two-dimensional substitution tilings have even slower growth, with the number of orientations growing only as the logarithm of the sample size. We expect that any three-dimensional substitution tiling that is qualitatively more isotropic than the QQ tiling would have to have a very complicated substitution rule. In other words, much of the complexity of the distribution of orientations would have to be built in from the start, sacrificing the elegant simplicity of the QQ tiling.

The orientations of a randomly chosen tile within the QQ tiling is described by a biased random walk in a dense subgroup of $S O$ (3). Lubotsky et al. [LPS1], [LPS2] considered unbiased random walks on such subgroups in their study of optimal distributions of points on $S^{2}$, and some of our methods are related to theirs. Long-time behavior of unbiased random walks on infinite cyclic subgroups of $S O(2)$ have been studied by $\mathrm{Su}[\mathrm{S}]$.

It is possible to compare the optimal distribution of elements of $S O(3)$ of [LPS1] with the distribution of orientations in the QQ tiling as follows. The "operator discrepancy" considered in [LPS1] is the operator norm of a certain Hecke operator acting on a subspace of $L^{2}(S O(3))$ (or $L^{2}\left(S^{2}\right)$ ). This operator is Hermitian and has discrete spectrum, so its operator norm is the supremum of the eigenvalues. For an optimal distribution of $N$ 
points, with $N-1$ prime, this supremum was shown to be $2 \sqrt{N-1} / N$, and in particular to decay as $N^{-1 / 2}$. In our case a distribution of orientations of $N$ QQ tiles is obtained by applying a fixed Hecke operator $\log _{8} N$ times; the $\log _{8} N$ th power of the largest eigenvalue of this Hecke operator goes as $N^{-1 / 336}$. These eigenvalues are discussed at greater length in Section 4.

The operator discrepancy is a very strict measure of anisotropy. Indeed, all distributions of points on the circle have operator discrepancy equal to 1 . In this paper we also consider two much weaker measures of spreading. The first is just a count of the number of distinct orientations in $S O(3)$, which turns out to scale as $N^{1 / 6}$. The second is the number of distinct orientations needed to account for half the sample, which scales as $N^{1 / 84}$. These measures are discussed in Sections 2 and 3, respectively.

The QQ tiling is constructed as follows (see [CR] for details). We begin with a single right triangular prism, $\sqrt{3}$ units wide, one unit high, and one unit deep, as in Fig. 2(a). This prism is divided into eight congruent pieces, each similar to the original. The front four resulting tiles are shown in Fig. 2(b), the back four in Fig. 2(c). We then rescale the entire pattern by a linear factor of 2 , obtaining a cluster of eight tiles, each congruent to the original.

We can repeat this process of subdivision and rescaling to obtain a cluster of 64 tiles, of 512 tiles, and so on. We embed each cluster of size $8^{n}$ within the succeeding cluster of size $8^{n+1}$ (there are eight ways to do this). The union of all the clusters is then an infinite tiling, typically covering all of $\mathbb{R}^{3}$.

After a "parent" tile is subdivided, the orientations of the "daughters" are closely related to that of the parent. We describe the orientation of a tile by a matrix $M \in$ $S O(3)$, the columns of which are unit vectors pointing along fixed features of the tile. There exist fixed matrices $g_{1}, \ldots, g_{8} \in S O(3)$ such that the orientation of the $i$ th daughter is $M g_{i}$, where the orientation of the parent is $M$. After two subdivisions, the orientations of the granddaughters are of the form $M g_{i} g_{j}$. After $n$ subdivisions the orientations of the descendant tiles are equal to $M$ times words of length $n$ in the generators $\left\{g_{i}\right\}$.

We now see why two-dimensional tilings have extremely slow growth. $S O(2)$ is abelian, so the number of words of length $n$ in a fixed set of generators has only polynomial growth in $n$, while the sample size of a substitution tiling is exponential in the number of subdivisions. In other words, the number of orientations is logarithmic in the sample size. $S O(3)$ is nonabelian, however, so the number of distinct words of length $n$ grows exponentially with $n$, hence as a power of the sample size. The power, however, need not be large. For the QQ tiling we will prove:

Theorem 1.1. Let $n \geq 2$. If $n=2 k$ is even, then the number of distinct orientations in the $n$th subdivision of a tile is $24\left(2^{k}-1\right)=24\left(N^{1 / 6}-1\right)$, where $N=8^{n}$ is the total number of tiles. If $n=2 k+1$ is odd, the number of distinct orientations in the $n$th subdivision is $34 \times 2^{k}-24=(34 / \sqrt{2}) N^{1 / 6}-24$.

By Theorem 1.1, a macroscopic sample of, say, $10^{24}$ tiles would appear in over 200,000 different orientations. The orientations do not, however, appear with equal frequency, and they are not evenly distributed in $S O(3)$. In a sample of $8^{27} \approx 2.4 \times 10^{24}$ tiles, a mere 104 orientations account for $69 \%$ of the tiles, while in a sample of $8^{120} \approx 2.3 \times 10^{108}$ 


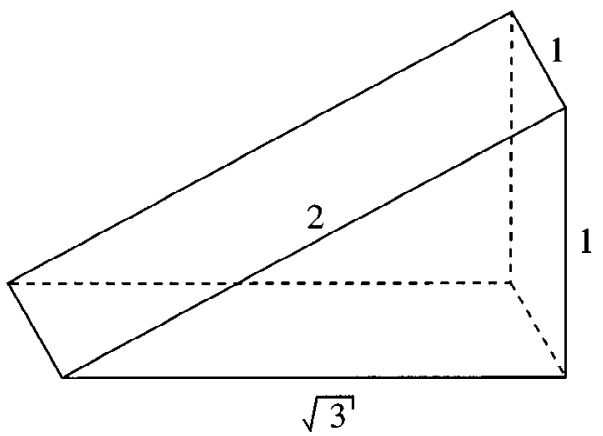

(a)

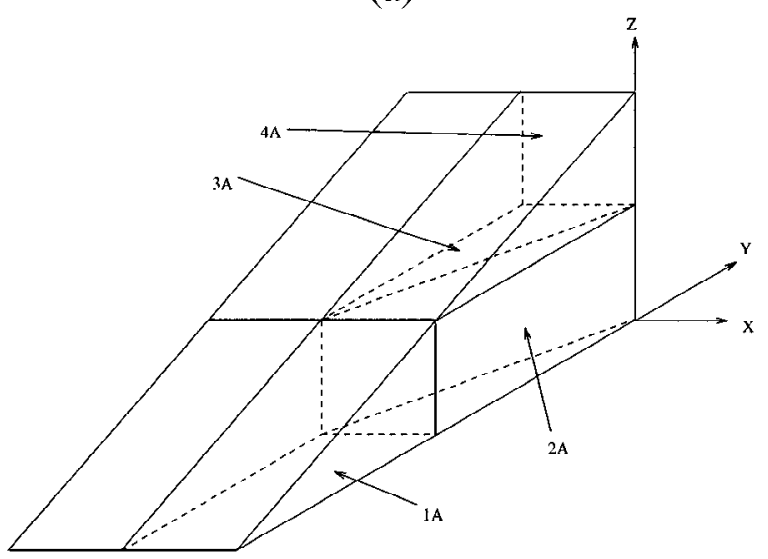

(b)

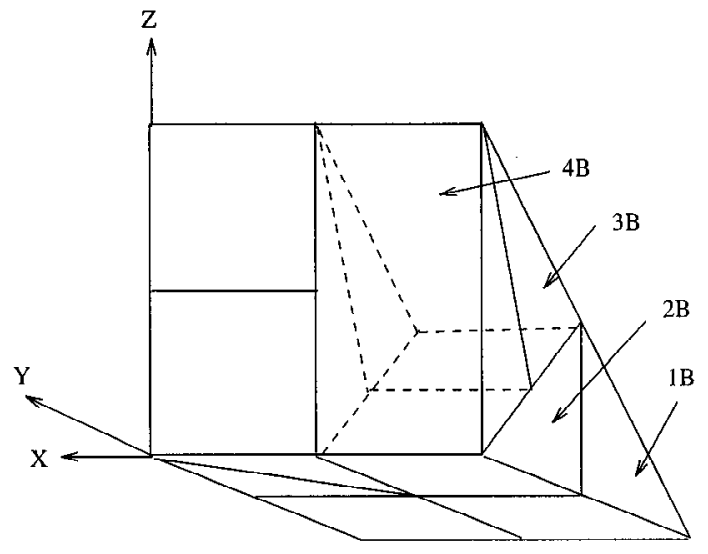

(c)

Fig. 2. A QQ tile (a) and the front (b) and the back (c) four daughter tiles. 
tiles, 1000 orientations account for slightly over half the tiles. The asymptotic behavior is given by:

Theorem 1.2. Let $\rho>\frac{1}{28}$, and let $F_{\alpha}(n)$ be the number of distinct orientations needed to account for a fraction $\alpha$ of the $8^{n}$ tiles in the $n$th subdivision. For any fixed $\alpha$,

$$
\lim _{n \rightarrow \infty} 2^{-\rho n} F_{\alpha}(n)=0 .
$$

That is, $2^{-n / 28} F_{\alpha}(n)$ is asymptotically smaller than any exponential in $n$ (i.e., any power of the volume), so we speak of $F_{\alpha}(n)$ as growing essentially as $2^{n / 28}$, or as the 84 th root of the volume.

Finally, we consider the rate at which the orientations approach a uniform distribution on $S O(3)$. Let $f(M)$ be a real-valued function of $M \in S O(3)$, and let $f_{n}(M)$ be the average value of $f$ on the $8^{n}$ orientations of the $n$ th-order descendants of a tile of orientation $M$. If $f$ is continuous, then $f_{n}(M)$ must converge to $\int_{S O(3)} f d \mu$ for every $M$, where $d \mu$ is the Haar measure on $S O(3)$. However, this convergence may be quite slow:

Theorem 1.3. There exists a smooth function $f: S O(3) \rightarrow \mathbb{R}$ and a point $M \in S O$ (3) such that $\left|f_{n}(M)-\int_{S O(3)} f d \mu\right|>(0.9938)^{n}$ for every $n$.

We believe this result to be close to sharp:

Conjecture 1.4. For any continuous function $f: S O(3) \rightarrow \mathbb{R}$ and any point $M \in$ $S O(3)$, there exists a constant $C$ such that $\left|f_{n}(M)-\int_{S O(3)} f d \mu\right|<C 2^{-n / 112} \approx$ $C(0.9938303)^{n}$ for every $n$.

In other words, it appears that functions do converge to their average values exponentially in the volume, with the exponents bounded away from zero, but there are exponents about as small as $\frac{1}{336}$. As in all linear systems of exponential decay, the long-time behavior is dominated by the smallest exponents, so we expect the anisotropy of a generic function on $S O$ (3) to decay roughly as $N^{-1 / 336}$ for sufficiently large $N$.

The outline of the paper is as follows. In Section 2 we examine the set of orientations that can arise from successive subdivisions of a single tile. This set turns out to be a subgroup, denoted $G(6,4)$, of $S O(3)$. The analysis of this section is a special case of some general results of [RS1]. We establish a canonical form for an element of $G(6,4)$ as a word in two generators, and provide an explicit list of the orientations in the $n$th subdivision, thereby proving Theorem 1.1 . We also develop a measure, called "size," of the complexity of an element of $G(6,4)$.

In Section 3 we consider the populations of the various orientations of the descendants of a given tile as a function of $n$, the number of subdivisions. We show that the "size" of the orientation of a randomly chosen tile is described by a biased random walk (on the nonnegative integers) with a reflecting barrier. Although the maximum size is $n / 2$, the distribution of sizes is approximately Gaussian with mean $n / 28+O(1)$. Since there are $O\left(2^{n / 28}\right)$ words of size $n / 28$ or less, Theorem 1.2 follows. 
Theorem 1.2 is an asymptotic statement. To obtain results for specific values of $n$ we numerically implement the random walk for $n$ up to 200. This establishes the claims previously made about samples of size $8^{27}$ and $8^{120}$.

In Section 4 we consider the rate at which the distribution of orientations approaches uniformity on $S O(3)$. There is a linear map $\mathcal{L}$ on $L^{2}(S O(3))$ such that $f_{n}=\mathcal{L}^{n} f$. $\mathcal{L}$ is similar to the Hecke operators considered in [LPS1] and [LPS2], except that those Hecke operators were Hermitian, while $\mathcal{L}$ is not. Nonetheless, the spectrum of $\mathcal{L}$ appears to be entirely real. If $f_{n}$ is to approach a limiting function exponentially, the base of the exponent (for generic $f$ ) is the largest eigenvalue of $\mathcal{L}$ that is less than 1 . The operator $\mathcal{L}$ is the direct sum of finite-dimensional blocks, each block corresponding to an irreducible representation of $S O(3)$. By numerically diagonalizing blocks corresponding to the first 300 representations (blocks with total dimension over $36,000,000$ ), we find a large number of eigenvalues over 0.993 , with the largest being approximately 0.99381 , and conjecture that the eigenvalues are bounded above by $2^{-1 / 112} \approx 0.9938303$.

\section{The Group $G(6,4)$}

Let $\mathbf{R}_{x}{ }^{\theta}$ denote a rotation about the $x$ axis by an angle $\theta$. The group generated by $\mathbf{R}_{x}{ }^{2 \pi / p}$ and $\mathbf{R}_{y}{ }^{2 \pi / q}$ is denoted $G(p, q)$. (For a discussion of $G(p, q)$ for general $p$ and $q$, and for further generalizations, see [RS1] and [RS2].) The set of orientations in the QQ tiling is precisely $G(6,4)$, generated by $S:=\mathbf{R}_{y}{ }^{\pi / 2}$ and $T:=\mathbf{R}_{x}{ }^{\pi / 3}$. Indeed, the orientations $g_{1}, \ldots, g_{8}$ of the eight daughters of a tile in standard position are all words in $S$ and $T$, and it is not hard to get $S$ and $T$ themselves as products of the $g_{i}$ 's. To understand these orientations, therefore, we must understand $G(6,4)$.

A word $\alpha$ in $S$ and $T$ is said to be in canonical form if

$$
\alpha=S^{a_{0}} T^{b_{0}}
$$

with $a_{0} \in\{0,1,2,3\}$ and $b_{0} \in\{0,3\}$, or if

$$
\alpha=W S T^{b_{1}} S T^{b_{2}} \cdots S T^{b_{n}} E
$$

with $n \geq 1$, with $W$ of the form (2.1), with each $b_{i}=2$ or 4 , and with $E=1$ or $S$. We shall soon see that each element of $G(6,4)$ can be expressed by a unique canonical form. The size of an element of $G(6,4)$ with canonical form (2.2) is defined to be $n$. (Elements of the form (2.1) have size zero.)

To understand the group $G(6,4)$, we represent $S$ and $T$ by explicit $3 \times 3$ matrices. Relative to the standard basis $\left\{e_{1}, e_{2}, e_{3}\right\}$ of $\mathbb{R}^{3}$, the matrices of $S$ and $T$ are

$$
S=\left(\begin{array}{ccc}
0 & 0 & 1 \\
0 & 1 & 0 \\
-1 & 0 & 0
\end{array}\right) \quad \text { and } \quad T=\left(\begin{array}{ccc}
1 & 0 & 0 \\
0 & 1 / 2 & -\sqrt{3} / 2 \\
0 & \sqrt{3} / 2 & 1 / 2
\end{array}\right)
$$

The factors of $\sqrt{3}$ are inconvenient to work with. We eliminate them by working in the 
basis $\left\{e_{1}, \sqrt{3} e_{2}, e_{3}\right\}$, relative to which the matrices of $S$ and $T$ are

$$
S=\left(\begin{array}{ccc}
0 & 0 & 1 \\
0 & 1 & 0 \\
-1 & 0 & 0
\end{array}\right), \quad T=\left(\begin{array}{ccc}
1 & 0 & 0 \\
0 & \frac{1}{2} & -\frac{1}{2} \\
0 & \frac{3}{2} & \frac{1}{2}
\end{array}\right)
$$

Three other useful matrices are

$$
T^{3}=\left(\begin{array}{ccc}
1 & 0 & 0 \\
0 & -1 & 0 \\
0 & 0 & -1
\end{array}\right), \quad S T^{ \pm 2}=\left(\begin{array}{ccc}
0 & \pm \frac{3}{2} & -\frac{1}{2} \\
0 & -\frac{1}{2} & \mp \frac{1}{2} \\
-1 & 0 & 0
\end{array}\right) .
$$

Theorem 2.1. Every element $g \in G(6,4)$ can be expressed in canonical form, and the canonical form is unique. If $g$ has size $n$, then, in the representation (2.4), $2^{n} g$ is an integer matrix with at least one odd matrix element. There exist exactly eight group elements of size 0 and $16 \times 2^{n}$ elements of size $n>0$.

Proof. Note that $2 S T^{2}$ and $2 S T^{4}$ are matrices with integer entries of the form

$$
\left(\begin{array}{lll}
\text { even } & \text { odd } & \text { odd } \\
\text { even } & \text { odd } & \text { odd } \\
\text { even } & \text { even } & \text { even }
\end{array}\right)
$$

and that the product of two or more matrices of the form given in (2.6) is also of the form given in (2.6). If $g$ can be expressed in canonical form (2.6), then

$$
\begin{aligned}
2^{n} g & =W\left(2 S T^{b_{1}}\right)\left(2 S T^{b_{2}}\right) \cdots\left(2 S T^{b_{n}}\right) E \\
& =W(\text { form } 2.6)(\text { form } 2.6) \cdots(\text { form } 2.6) E \\
& =W(\text { form } 2.6) E .
\end{aligned}
$$

Since $S$ is a permutation matrix (up to sign), and $T^{3}$ is the identity (up to sign), $2^{n} g$ is an integer matrix with four odd matrix elements and five even matrix elements. If $g$ is of canonical form (2.1), then $2^{n} g=2^{0} g=g$ is an integer matrix with three odd matrix elements. This proves the second statement of the theorem, and also shows that the identity can be put in canonical form in only one way, namely $1=S^{0} T^{0}$.

To see that every element of $G(6,4)$ can be put in canonical form, begin with an arbitrary word

$$
\alpha=S^{a_{1}} T^{b_{1}} \cdots S^{a_{n}} T^{b_{n}}
$$

in $S$ and $T$. If any of the $a_{i}$ 's is even, or any of the $b_{i}$ 's is a multiple of 3 , we can use the relations

$$
S T^{3}=T^{3} S^{-1}
$$

and

$$
S^{2} T=T^{-1} S^{2}
$$

to shorten the word. So we can assume that all the $a_{i}$ 's (except possibly $a_{1}$ ) are odd, and that none of the $b_{i}$ 's (except possibly $b_{1}$ ) are multiples of 3 . We then use (2.9) to 
extract powers of $T^{3}$ and move them leftwards, changing $T^{1}$ to $T^{4}$ and changing $T^{5}$ to $T^{2}$. We then use (2.10) to extract powers of $S^{2}$ and move them leftwards, changing $S^{3}$ to $S$. In the process, we may change some $T^{2}$ 's to $T^{4}$ 's and vice versa, but that does not affect the form (2.2). Finally, if $b_{1}$ is not divisible by 3, we use (2.9) to rewrite $S^{a_{1}} T^{b_{1}}$ as $S^{a_{0}} T^{3 b_{0}} S T^{b_{1}^{\prime}}$ with $b_{1}^{\prime}=2$ or 4 .

To see that a canonical form is unique, suppose $g$ and $g^{\prime}$ are distinct canonical forms for the same group element. Using the relations (2.9) and (2.10), we could then shorten the word $g^{\prime} g^{-1}$ to a nontrivial word in canonical form, which would have to represent the identity. However, we have already seen that there are no nontrivial canonical forms for the identity.

Since group elements are in 1-1 correspondence with canonical forms, we can count group elements of any given size. There are eight choices for $W$, two choices for each $b_{i}$, and two choices for $E$, for a total of $16 \times 2^{n}$ elements of size $n>0$. For size 0 there are only eight choices, four for $a_{0}$ and two for $b_{0}$.

We now have the machinery to understand the orientations of tiles in the $n$-fold subdivision of a single QQ tile. In a single subdivision, the daughters are described by group elements $\left\{g_{1}, \ldots, g_{8}\right\}$ with

$$
\begin{aligned}
& g_{1}=\mathbf{R}_{x}{ }^{0}=1, \\
& g_{2}=\mathbf{R}_{y}{ }^{3 \pi / 2} \mathbf{R}_{z}{ }^{\pi}=S T^{3} \text {, } \\
& g_{3}=\mathbf{R}_{y}{ }^{\pi / 2}=S \text {, } \\
& g_{4}=\mathbf{R}_{x}^{0}=1 \text {, } \\
& g_{5}=\mathbf{R}_{x}^{0}=1 \text {, } \\
& g_{6}=\mathbf{R}_{z}{ }^{\pi}=S^{2} T^{3} \text {, } \\
& g_{7}=\mathbf{R}_{x}{ }^{4 \pi / 3} \mathbf{R}_{y}{ }^{\pi}=T^{4} S^{2}=S^{2} T^{2} \text {, } \\
& g_{8}=\mathbf{R}_{x}^{4 \pi / 3}=T^{4} \text {. }
\end{aligned}
$$

Note that $T=g_{7} g_{6}$, so the semigroup generated by the $g_{i}$ 's is precisely the group generated by $S$ and $T$. Also note that only two of the $g_{i}$ 's, namely $g_{7}$ and $g_{8}$, have size 1 , while the rest have size 0 . Since $g_{7}^{2}, g_{8}^{2}, g_{7} g_{8}$, and $g_{8} g_{7}$ all have size 1 or less, the orientations in the second subdivision have size at most 1 and the orientations in the $2 k$ th subdivision have size at most $k$. This shows that the number of possible orientations in the $n$th subdivision is bounded by a constant multiple of $2^{n / 2}$.

However, we can be more precise. The following theorem implies Theorem 1.1:

Theorem 2.2. The orientations appearing in subdivisions of a single tile (of standard orientation) are as follows. Let $P_{m}$ be the set of words of length $m$ in the two generators $S T^{2}$ and $S T^{4}$. For $k>0$, the orientations of the $2 k$ th subdivision are:

1. $T^{b} P_{m} S^{a}$, with no restriction on a or $b$, and with $m$ ranging from 0 to $k-2$,

2. $T^{b} P_{k-1} S^{a}$, with $b=0,2,3$, or 5 and a arbitrary,

3. $T^{b} P_{k-1} S^{a}$, with $b=1$ or 4 and $a=0$ or 2 ,

4. $S T^{2} P_{k-1} S^{a}$, with $a=0$ or 2 , and

5. $T^{3}\left(S T^{4}\right) P_{k-1} S^{a}$, with $a=0$ or 2 . 
For $k>0$, the orientations in the $(2 k+1)$ st subdivision are

1. $T^{b} P_{m} S^{a}$, with no restriction on $a$ or $b$ and $m$ ranging from 0 to $k-1$,

2. $T^{b} P_{k} S^{a}$, with $b=0,2,3$, or 5 and $a=0$ or 2 ,

3. $S T^{2} P_{k-1} S^{a}$, with $a=1$ or 3 , and

4. $T^{3} S T^{4} P_{k-1} S^{a}$, with $a=1$ or 3 .

Proof. It is not hard to see that, in each case, the sets are disjoint and their cardinalities add up to the total indicated in Theorem 1.1. To complete the proof we must check (i) that the claimed orientations for two subdivisions are correct, (ii) that the claimed orientations for $2 k$ subdivisions times the generators $g_{1}, \ldots, g_{8}$ give precisely the claimed orientations for $2 k+1$ subdivisions, and (iii) that the claimed orientations for $2 k+1$ subdivisions times the generators $g_{1}, \ldots, g_{8}$ give precisely the claimed orientations for $2 k+2$ subdivisions. We leave this straightforward (but quite tedious) computation as an exercise for the highly motivated reader.

\section{Population Dynamics}

In Section 2 we counted the number of distinct orientations of tiles in the $n$th subdivision of a QQ tile, which equals the number of algebraically distinct words of length $n$ in the generators $g_{1}, \ldots, g_{8}$. In this section we consider the distribution of tiles among the various orientations. Equivalently, we count repetitions in the words of length $n$ in the $g_{i}$ 's.

We do this by modeling the subdivision process as a random walk on $G(6,4)$. If at some stage we are at the group element $M$, then at the next stage we can go to $M g_{1}, M g_{2}, \ldots, M g_{8}$, each with probability $1 / 8$. The distribution of orientations in the $n$th subdivision of a tile (with standard orientation) is precisely the $n$th iterate of this random walk, starting at the origin. The following theorem implies Theorem 1.2.

Theorem 3.1. Consider the random walk starting at the origin, and let $P(k, n)$ be the probability that the $n$th iteration yields a group element of size $k$ or greater. For any $\rho>\frac{1}{28}, \lim _{n \rightarrow \infty} P(n \rho, n)=0$.

Proof. We divide $G(6,4)$ into sets according to size and the pattern at the end of the canonical form. The random walk on $G(6,4)$ then induces a random walk on the space of sets, and we show that the bias in this random walk causes the average size to increase by $\frac{1}{28}$ each turn. The result then follows from the central limit theorem.

Let $R\left(k, S T^{2}\right)$ be the set of group elements of size $k$ whose canonical forms end with $S T^{2} . R\left(k, S T^{4}\right), R\left(k, T^{2} S\right)$, and $R\left(k, T^{4} S\right)$ are defined similarly. $R(0)$ is the set of elements of size 0 .

If $M \in R\left(k, S T^{2}\right)$, then $M g_{1}, M g_{4}$ and $M g_{5}$ are equal to $M$, and so are in $R\left(k, S T^{2}\right)$. $M g_{2}$ is in $R\left(k, T^{4} S\right), M g_{3}$ is in $R\left(k, T^{2} S\right)$, and $M g_{6}$ is in $R\left(k, S T^{4}\right) . M g_{7}$ may be in $R\left(k-1, T^{2} S\right)$ or $R\left(k-1, T^{4} S\right)$, depending on whether $M$ ends with $S T^{2} S T^{2}$ or $S T^{4} S T^{2}$. However, if $M g_{7} \in R\left(k-1, T^{2} S\right)$, then $M g_{8} \in R\left(k-1, T^{4} S\right)$, and vice versa. In either case, starting in $R\left(k, S T^{2}\right)$, there is a $3 / 8$ probability of staying in $R\left(k, S T^{2}\right)$ 
and a $1 / 8$ probabilty for each of the destinations $R\left(k, S T^{4}\right), R\left(k, T^{2} S\right), R\left(k, T^{4} S\right)$, $R\left(k-1, T^{2} S\right)$ and $R\left(k-1, T^{4} S\right)$.

This analysis, and the corresponding analysis for $R\left(k, S T^{4}\right), R\left(k, T^{2} S\right)$, and $R\left(k, T^{4} S\right)$, is summarized in the diagram

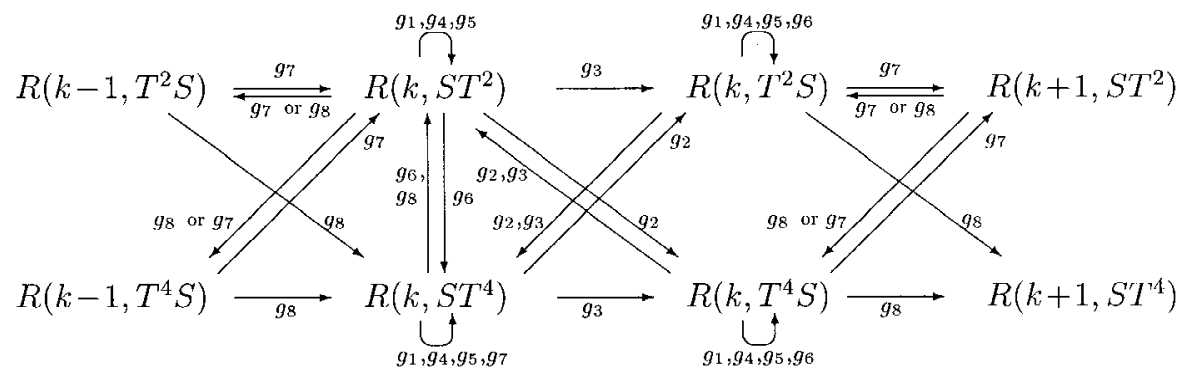

This diagram continues to the right indefinitely, but to the left it ends at $k=0$ with

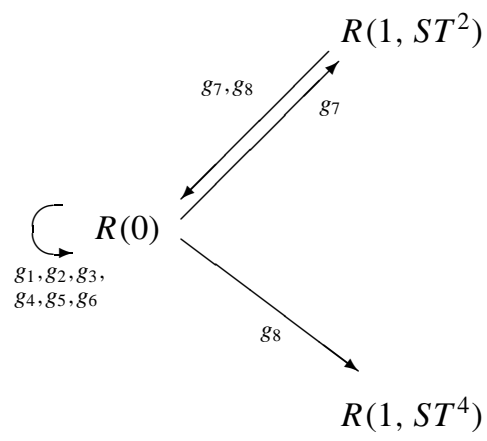

Since the long-time behaviors of positively biased random walks are independent of the boundary conditions at $k=0$, the reflecting barrier at $k=0$ does not affect the theorem. To establish the theorem, we need only calculate the bias in the random walk away from $k=0$ and show it equals $\frac{1}{28}$.

We first compute the distribution of $S T^{2}, S T^{4}, T^{2} S$ and $T^{4} S$ endings. Summing over $k$, we find the transition matrix for the four classes of group elements is

$$
\frac{1}{8}\left(\begin{array}{llll}
3 & 2 & 1 & 3 \\
1 & 4 & 3 & 1 \\
2 & 1 & 4 & 0 \\
2 & 1 & 0 & 4
\end{array}\right)
$$

The limiting distribution is the eigenvector with eigenvalue 1, namely

$$
\frac{1}{14}\left(\begin{array}{l}
4 \\
4 \\
3 \\
3
\end{array}\right) \text {. }
$$


That is, asymptotically $\frac{4}{14}$ of the tiles have orientations whose canonical forms end with $S T^{2}, \frac{4}{14}$ end with $S T^{4}, \frac{3}{14}$ end with $T^{2} S$, and $\frac{3}{14}$ end with $T^{4} S$.

On each iteration, an $S T^{2}$ state has a $\frac{2}{8}$ chance of decreasing $k$ (and none of increasing), $S T^{4}$ states have no chance of either increasing or decreasing $k$, while $T^{2} S$ and $T^{4} S$ states each have a $\frac{2}{8}$ chance of increasing $k$ and no chance of decreasing. Weighing these chances by the $\left(\frac{4}{14}, \frac{4}{14}, \frac{3}{14}, \frac{3}{14}\right)$ limiting distribution, we see that at each step in the random walk there is a $\frac{3}{28}$ chance of increasing $k$ and a $\frac{2}{28}$ chance of decreasing $k$, for a net bias of $\frac{1}{28}$.

Theorem 3.1 is an asymptotic result that does not indicate how many iterations are needed to reach the asymptotic regime. Indeed, the bias in the random walk is so small that, for moderate numbers of iterations, one would expect diffusion and the reflecting barrier at $k=0$ to be quite significant. To measure this transient behavior we rely on the following numerical implementation, in MATLAB, of the biased random walk. Here $z z(n)$ is the fraction of orientations of size 0 in the $n$th generation, st $2(k, n)$ (resp. $\mathrm{st} 4(\mathrm{k}, \mathrm{n}), \mathrm{t} 2 \mathrm{~s}(\mathrm{k}, \mathrm{n}), \mathrm{t} 4 \mathrm{~s}(\mathrm{k}, \mathrm{n}))$ is the fraction in $R\left(S T^{2}, k\right)\left(\operatorname{resp} . R\left(S T^{4}, k\right)\right.$, $\left.R\left(T^{2} S, k\right), R\left(T^{4} S, k\right)\right)$ in the $n$th generation, and tot $(\mathrm{k}+1, \mathrm{n})$ is the fraction of size $k$ in the $n$th generation.

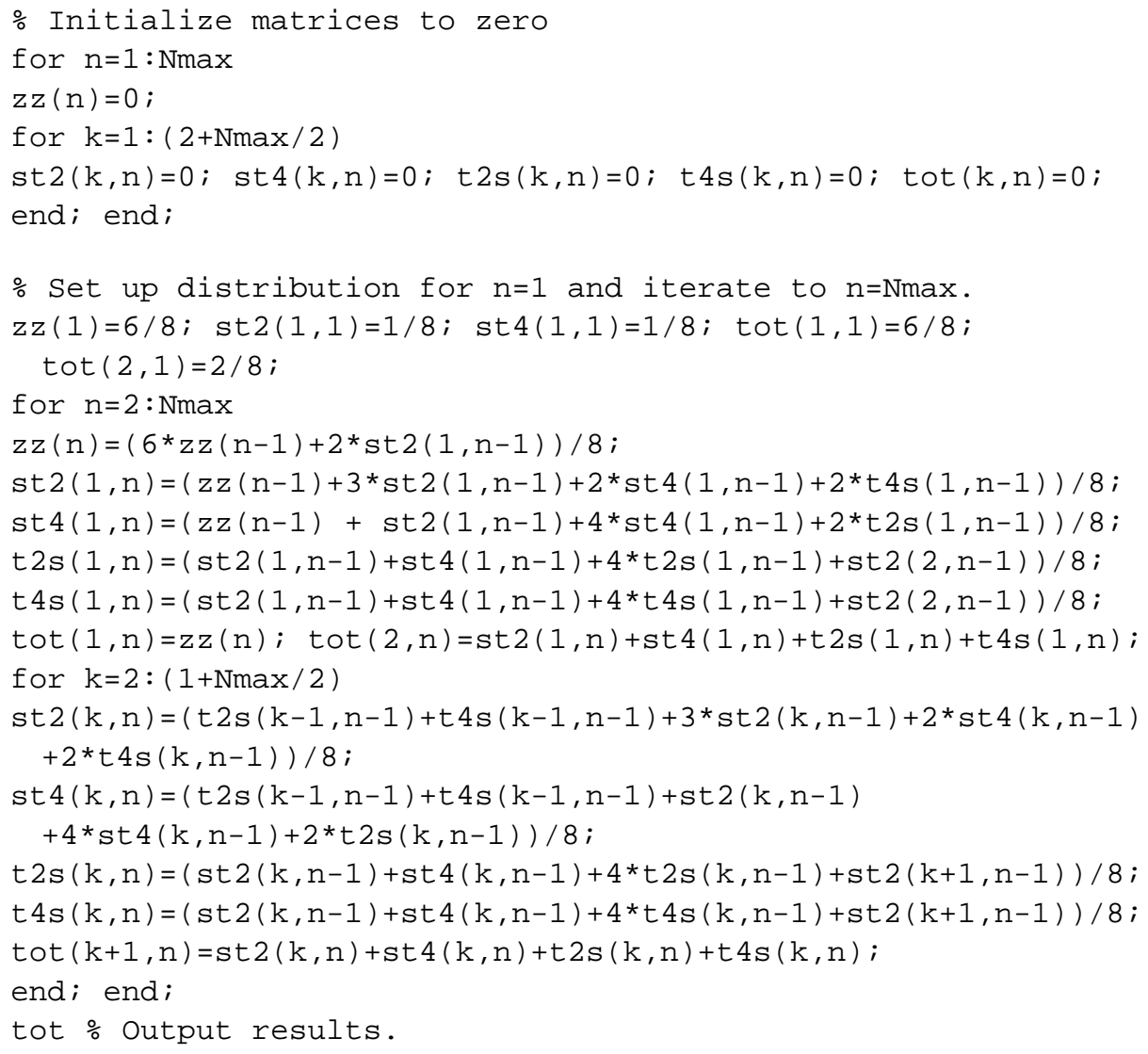


Running this program with $\operatorname{Nmax}=200$, one sees that the median orientation size is

$$
\text { median size }=\left\{\begin{array}{lll}
0 & \text { if } \quad n \leq 2 ; \\
1 & \text { if } \quad 2<n \leq 20 \\
2 & \text { if } \quad 20<n \leq 42 \\
3 & \text { if } \quad 42<n \leq 67 \\
4 & \text { if } \quad 67<n \leq 93 \\
5 & \text { if } \quad 93<n \leq 120 \\
6 & \text { if } \quad 120<n \leq 147 \\
7 & \text { if } \quad 147<n \leq 174 \\
8 & \text { if } \quad S 174<n \leq 200
\end{array}\right.
$$

Note that by Theorem 2.1 there are exactly 8 orientations of size 0,40 of size at most 1,104 of size at most 2, 232 of size at most 3,488 of size at most 4, 1000 of size at most 5,2024 of size at most 6,4072 of size at most 7, and 8168 of size at most 8 . Thus for $n=27$, just 104 orientations account for the majority of the tiles, while for $n=120$, a mere 1000 orientations account for the majority of the tiles.

Table 1 shows the distribution of sizes for three samples, a microscopic sample with $8^{5}=32,768$ tiles, a macroscopic sample with $8^{27} \approx 2.42 \times 10^{24}$ tiles (about 4 moles), and an impossibly large sample of $8^{120} \approx 2.35 \times 10^{108}$ tiles. Table 2 shows similar results for $n=10,20,30,40$, and 50 .

Table 1. Distributions of sizes in various samples.

\begin{tabular}{cllc}
\hline Size & $n=8$ & $n=27$ & $n=120$ \\
\hline 0 & 0.2588 & 0.0971 & 0.0145 \\
1 & 0.5531 & 0.3023 & 0.0524 \\
2 & 0.1719 & 0.2931 & 0.0788 \\
3 & 0.0159 & 0.1903 & 0.1038 \\
4 & 0.0003 & 0.0847 & 0.1221 \\
5 & 0 & 0.0260 & 0.1299 \\
6 & 0 & 0.0055 & 0.1256 \\
7 & 0 & 0.0008 & 0.1110 \\
8 & 0 & 0.0001 & 0.0897 \\
9 & 0 & 0 & 0.0665 \\
10 & 0 & 0 & 0.0453 \\
11 & 0 & 0 & 0.0283 \\
12 & 0 & 0 & 0.0163 \\
13 & 0 & 0 & 0.0086 \\
14 & 0 & 0 & 0.0042 \\
15 & 0 & 0 & 0.0019 \\
16 & 0 & 0 & 0.0008 \\
17 & 0 & 0 & 0.0003 \\
18 & 0 & 0 & 0.0001 \\
19 & 0 & 0 & 0.0000 \\
\hline
\end{tabular}


Table 2. Additional distributions of sizes.

\begin{tabular}{rlllll}
\hline Size & $n=10$ & $n=20$ & $n=30$ & $n=40$ & $n=50$ \\
\hline 0 & 0.2199 & 0.1272 & 0.0878 & 0.0654 & 0.0508 \\
1 & 0.5230 & 0.3738 & 0.2780 & 0.2154 & 0.1718 \\
2 & 0.2199 & 0.3023 & 0.2842 & 0.2488 & 0.2140 \\
3 & 0.0353 & 0.1457 & 0.2013 & 0.2149 & 0.2089 \\
4 & 0.0019 & 0.0426 & 0.1011 & 0.1422 & 0.1635 \\
5 & 0.0000 & 0.0075 & 0.0363 & 0.0728 & 0.1038 \\
6 & 0 & 0.0008 & 0.0093 & 0.0290 & 0.0537 \\
7 & 0 & 0.0000 & 0.0017 & 0.0090 & 0.0227 \\
8 & 0 & 0.0000 & 0.0002 & 0.0021 & 0.0079 \\
9 & 0 & 0.0000 & 0.0000 & 0.0004 & 0.0022 \\
10 & 0 & 0.0000 & 0.0000 & 0.0001 & 0.0005 \\
\hline
\end{tabular}

\section{Approach to the Ergodic Limit}

Let $f(M)$ be a function on $S O(3)$ and let $f_{n}(M)$ be the average of $f$ over the $8^{n}$ tiles in the $n$-fold subdivision of a tile of orientation $M$. Equivalently,

$$
f_{n}(M)=8^{-n} \sum_{i_{1}=1}^{8} \sum_{i_{2}=1}^{8} \cdots \sum_{i_{n}=1}^{8} f\left(M g_{i_{1}} g_{i_{2}} \cdots g_{i_{n}}\right) \text {. }
$$

As $n \rightarrow \infty, f_{n}(g)$ approaches the constant limit $\int_{S O(3)} f d \mu$, where $d \mu$ is the Haar measure. The question is how fast the (ergodic) limit is approached.

One approach to the problem is to consider the linear operator $\mathcal{L}: L^{2}(S O(3)) \rightarrow$ $L^{2}(S O(3))$ defined by

$$
\mathcal{L} f(M)=\frac{1}{8} \sum_{i=1}^{8} f\left(M g_{i}\right)
$$

We can expand an arbitrary function in eigenfunctions $\xi_{i}$ of $\mathcal{L}$, with eigenvalues $\lambda_{i}$ :

$$
f=\sum_{i} a_{i} \xi_{i}
$$

so that

$$
f_{n}=\sum_{i} a_{i} \lambda_{i}^{n} \xi_{i}
$$

Of course, the constant function is an eigenfunction of $\mathcal{L}$ with eigenvalue 1 . The rate at which $f_{n}$ approaches a constant function is determined by the second-largest eigenvalue of $\mathcal{L}$. 
The operator $\mathcal{L}$ is quite similar to the Hecke operators considered in [LPS1] and [LPS2], except that the set $\left\{g_{i}\right\}$ is not balanced, as the inverses of $g_{3}$ and $g_{8}$ are not in the set. As a result, $\mathcal{L}$ is not a self-adjoint operator on $L^{2}(S O(3))$, not even with a weighted measure, and we know of no a priori reason why the eigenvalues of $\mathcal{L}$ need be real. However, the numerical experiments described below indicate that the eigenvalues of $\mathcal{L}$ are indeed real. We do not understand the reason for this.

The natural (left and right) actions of $S O(3)$ on $L^{2}(S O(3))$, namely $(l, r): f(g) \rightarrow$ $f(\lg r)$, decomposes $L^{2}(S O(3))$ into irreducible representations of $S O(3)$. Since $\mathcal{L}$ is a linear combination of right-translations, it acts separately on each representation. Thus the infinite-dimensional job of diagonalizing $\mathcal{L}$ on all of $L^{2}$ breaks down into a sequence of finite-dimensional problems, namely diagonalizing $\mathcal{L}$ on each irreducible representation that appears in the decomposition of $L^{2}$.

The irreducible representations of $S O(3)$ are standard. The spin- $\ell$ representation, of dimension $2 \ell+1$, appears $2 \ell+1$ times in the decomposition of $L^{2}$. In particular, each representation appears at least once. For each representation $R_{\ell}$, we must diagonalize the $(2 \ell+1) \times(2 \ell+1)$ matrix

$$
\mathcal{L}_{\ell}=\frac{1}{8}\left[R_{\ell}\left(g_{1}\right)+\cdots+R_{\ell}\left(g_{8}\right)\right]
$$

We have done this, using MATLAB, for $\ell$ up to 300 , thereby diagonalizing $\mathcal{L}$ on a subspace of $L^{2}(S O(3))$ of total dimension greater than 36,000,000. There are numerous eigenvalues over 0.993 , but none over 0.994; the largest known eigenvalue is $\lambda \approx 0.99381$, appearing in the $\ell=258$ representation. This proves Theorem 1.4, and suggests there is an upper bound to the eigenvalues slightly above 0.99381 . Since powers of 2 appear repeatedly in the analysis, and since Theorem 1.2 suggests a time scale of 28 subdivisions, $2^{-1 /(4 \times 28)} \approx 0.9938303$ is a fairly natural guess for that upper bound.

Table 3 lists the largest eigenvalue for each of the first 20 representations. Table 4 lists the "record" eigenvalues, those larger than any eigenvalue corresponding to smaller $\ell$. The

Table 3. Largest eigenvalues for first 20 representations.

\begin{tabular}{rccc}
\hline$\ell$ & Eigenvalue & $\ell$ & Eigenvalue \\
\hline 1 & 0.50000 & 11 & 0.90142 \\
2 & 0.78785 & 12 & 0.96793 \\
3 & 0.50000 & 13 & 0.94705 \\
4 & 0.92693 & 14 & 0.98797 \\
5 & 0.62500 & 15 & 0.90546 \\
6 & 0.94912 & 16 & 0.96437 \\
7 & 0.84548 & 17 & 0.94949 \\
8 & 0.98454 & 18 & 0.99048 \\
9 & 0.73649 & 19 & 0.93382 \\
10 & 0.95848 & 20 & 0.98042
\end{tabular}


Table 4. Record eigenvalues.

\begin{tabular}{rc}
\hline$\ell$ & Eigenvalue \\
\hline 1 & 0.50000 \\
2 & 0.78785 \\
4 & 0.92693 \\
6 & 0.94912 \\
8 & 0.98454 \\
14 & 0.98797 \\
18 & 0.99048 \\
32 & 0.99243 \\
45 & 0.99324 \\
56 & 0.99335 \\
72 & 0.99362 \\
248 & 0.99367 \\
258 & 0.99381 \\
\hline
\end{tabular}

MATLAB program for finding the largest eigenvalues from the first Lmax representations is

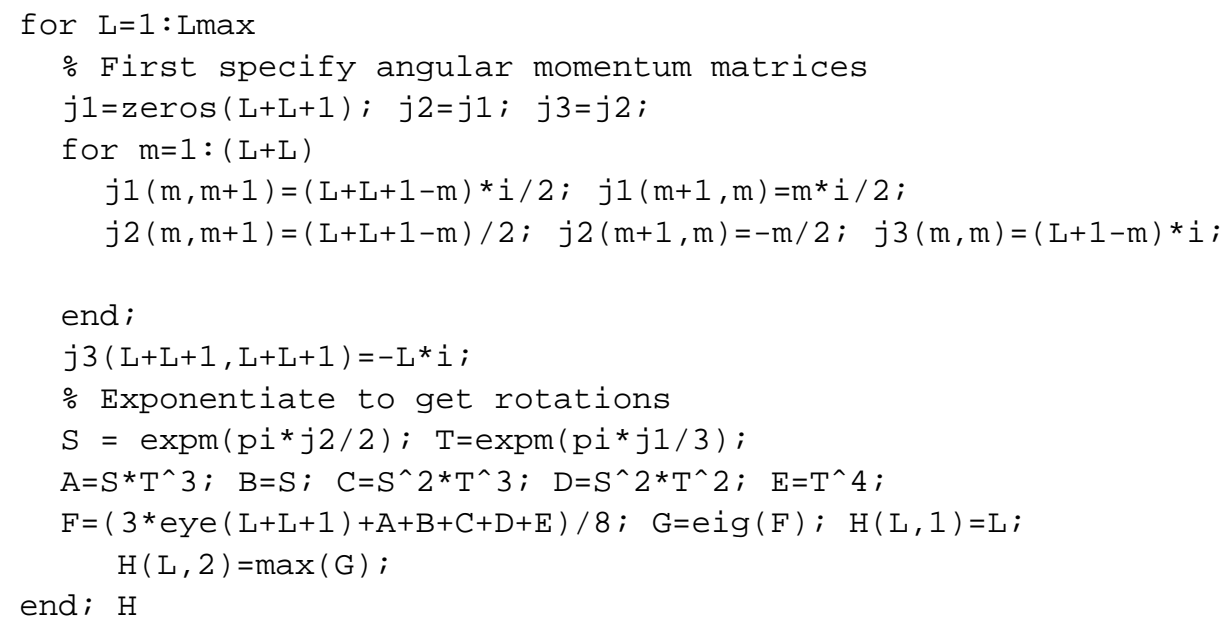

\section{Conclusions and Speculations}

Much of the interest in tilings stems from the fact that crystals and quasicrystals are modeled by tilings in which the tiles appear in only a finite number of orientations. Is there a form of matter that is modeled on aperiodic tilings with statistical rotational symmetry, in particular on a substitution tiling such as the QQ? If so, how would such a form of matter be recognized?

This paper provides the tools needed to recognize such a substance. We have computed the distribution of orientations in a sample of size $N=8^{n}$ of the QQ tiling. The exact same calculation gives the distribution of orientations of 8-tile clusters in a 
sample of size $8^{n+1}$, of 64 -tile clusters in a sample of size $8^{n+2}$, and so on. In particular, the angular distribution of the relative positions of nearby tiles, and therefore the angular distribution of the electron diffraction pattern, are governed by the results of this paper.

To detect an asymptotically round quasicrystal, one must look at the diffraction pattern of a finite sample and decompose it into spherical harmonics. As long as the wavelengths in question are much shorter than the size of the sample, the angular distribution of the diffraction pattern will scale with sample size in a manner determined by the eigenvalues. If a spherical harmonic is an eigenvector of $\mathcal{L}$ with eigenvalue $\lambda$, then the diffraction pattern of a sample of size $8 N$ will exhibit this harmonic a factor of $\lambda$ less than that of a sample of size $N$.

As a result, some harmonics will be essentially absent from the diffraction pattern of a macroscopic sample; those correspond to eigenfunctions of $\mathcal{L}$ with $\lambda^{n} \ll 1$. Other modes, with $1-\lambda$ comparable with or less than $1 / n$, will appear. A macroscopic (say, $N=8^{27}$ ) sample of the QQ tiling, for example, would have a diffraction pattern with a small but probably detectable $\ell=4$ component, and with essentially undamped $\ell=6$, $\ell=8$, and $\ell=10$ components. There are relatively few eigenfunctions with such large eigenvalues (even for $\ell=8$, all but two of the 17 eigenvalues are less than 0.65 ), so the diffraction pattern will be dominated by the $\ell=0$ (isotropic) component, with some corrections from harmonics with $\ell=6,8,10$, etc. This result is much more isotropic than that of a crystal or ordinary quasicrystal (for which many harmonics are completely undamped, and the spectrum consists of discrete points), while much less isotropic than that of an amorphous substance such as a glass, for which only the isotropic $\ell=0$ term is observable.

The radial dependence of the diffraction pattern also carries important information. As with other quasicrystals, and unlike amorphous media, one expects asymptotically round quasicrystals to have radially self-similar diffraction patterns. In combination, a self-similar radial pattern combined with a mildly anisotropic angular pattern should indicate an asymptotically round quasicrystal.

\section{Acknowledgments}

We thank Charles Radin for helpful discussions and Margaret Combs for her TEXnical expertise. B. D. thanks J. M. Linhart for her guidance and support.

\section{References}

[CR] J. Conway and C. Radin. Quaquaversal tilings and rotations. Invent. Math. 132 (1998), 179188.

[LPS1] A. Lubotsky, R. Phillips, and P. Sarnak. Hecke operators and distributing points on the sphere, I. Comm. Pure Appl. Math. 39 (1986) S149-S186.

[LPS2] A. Lubotsky, R. Phillips, and P. Sarnak. Hecke operators and distributing points on $S^{2}$, II. Comm. Pure Appl. Math. 40 (1987) 401-420. 
[R1] C. Radin. Symmetry and tilings. Notices Amer. Math. Soc. 42 (1995), 26-31.

[R2] C. Radin. Private communication.

[RS1] C. Radin and L. Sadun. Subgroups of SO(3) associated with tilings. J. Algebra 202 (1998), 611-633.

[RS2] C. Radin and L. Sadun. On 2-generator subgroups of SO(3). Trans. Amer. Math. Soc., 351 (1999), 4469-4480.

[S] F. Su, Convergence of random walks on the circle generated by an irrational rotation. Trans. Amer. Math. Soc. 350 (1998), 3717-3741.

Received October 19, 1998, and in revised form March 11, 1999. 